\title{
Correlation between interleukin 4 (IL-4) expression and allergen sensitization in allergic rhinitis patients
}

\section{Correlación entre la expresión de interleucina 4 y la sensibilización a alérgenos en pa- cientes con rinitis alérgica}

Asti Widuri ${ }^{1}$

\begin{abstract}
Objective: The objective of this research was to measure the correlation of the expression of IL-4 with allergen sensitization in patients with allergic rhinitis.

Methods: Descriptive analytics was used as a method in this research, with a cross-sectional approach. The subjects were 37 persons with allergic rhinitis who met the inclusion and exclusion criteria. This research was conducted in patients with clinical manifestations of symptoms of allergic rhinitis, and skin prick tests were carried out with ten types of allergens, which are: house dust, cotton, chicken feathers, shrimp, tuna, chicken egg-yolk, chicken egg-white, peanuts, tea, and chocolate. Venous blood sampling was performed for the Interleukin-4 (IL-4) test by using an ELISA device.

Results: The respondents were 37 patients with allergic rhinitis, of which 26 (70.3\%) were women and 11 (29.7 \%) were men. The highest sensitivity to inhaled allergens in patients with rhinitis was to house dust in 31 samples ( $83.8 \%$ ). The correlation of the expression of IL-4 with sensitization to allergens in patients with allergic rhinitis based on the Spearman test resulted in $p>0.05$, not significant with some allergens in a negative correlation.

Conclusion: There was no significant correlation between the expression of IL-4 and the sensitization to allergens in patients with allergic rhinitis.
\end{abstract}

Key words: Interleukin-4; Allergic rhinitis; Allergen; Sensitization

\section{Resumen}

Objetivo: Esta investigación tuvo como objetivo medir la correlación de la expresión de IL-4 con la sensibilización a alérgenos en pacientes con rinitis alérgica.

Métodos: Esta investigación utilizó la analítica descriptiva como método, con un enfoque transversal. Los sujetos fueron 37 personas con rinitis alérgica que cumplieron con los criterios de inclusión y exclusión. Esta investigación se realizó en pacientes con manifestaciones clínicas de síntomas de rinitis alérgica y prueba de punción cutánea mediante la prueba de diez tipos de alérgenos, estos son: polvo doméstico, algodón, plumas de pollo, camarones, atún, yema de huevo de gallina, clara de huevo de gallina, maní, té, y chocolate. Se realizó una muestra de sangre venosa para el examen de interleucina-4 (IL-4) utilizando un dispositivo ELISA.

Resultados: Los encuestados fueron 37 pacientes con rinitis alérgica divididos en 26 (70,3 \%) mujeres y 11 (29,7 \%) hombres. La mayor sensibilidad a alérgenos inhalados en pacientes con rinitis fue el polvo doméstico en 31 muestras (83,8 \%). La correlación de la expresión de IL-4 con la sensibilización a alérgenos en pacientes con rinitis alérgica basada en la prueba de Spearman se obtuvo p > 0,05, no significativa con algunos alérgenos en correlación negativa.

Conclusión: No hubo correlación significativa entre la expresión de IL-4 y la sensibilización a alérgenos en pacientes con rinitis alérgica.

Palabras clave: Interleucina-4; Rinitis alérgica; Alérgeno; Sensibilización

${ }^{1}$ Universidad Muhammadiyah Yogyakarta, Facultad de Medicina y Ciencias de la Salud, Departamento de Otorrinolaringología, Yogyakarta, Indonesia
Correspondencia: Asti Widuri. astiwiduri@gmail.com

Recibido: 2020-08-25

Aceptado: 2021-04-23 DOI: $10.29262 / \mathrm{ram} . v 658 \mathrm{i} 2.800$ 


\section{Introducción}

Las enfermedades alérgicas del tracto respiratorio, incluyendo asma y la rinitis alérgica (RA), son problemas de salud mundial con una alta incidencia que afectan la calidad de vida y la economía. ${ }^{1,2} \mathrm{El}$ manejo de las alergias respiratorias evitando los alérgenos desencadenantes, ${ }^{3}$ medicamentos con antihistamínicos, corticosteroides nasales y leucotrienos e inmunoterapia para los casos graves que fracasan médicamente, o evitando la terapia con medicamentos a largo plazo. ${ }^{4}$

La RA es una enfermedad inflamatoria alérgica de la mucosa nasal, caracterizada por una respuesta inmunitaria IgE. Las células T helper (Th) 2 desempeñan un papel importante en el desarrollo de enfermedades mediadas por inmunoglobulina E (IgE), como la RA, al producir en exceso citocinas Th2 locales (interleucinas [IL] 4, IL-5 e IL-13) en los sitios de inflamación alérgica. ${ }^{5}$ Se sabe que el equilibrio de citocinas Th1 (IL-12 e interferón gamma) suprime esta respuesta inmunitaria Th2, y la citocina Th2 (IL-4) ayuda a tratar esta enfermedad. El conocimiento de los mecanismos inmunológicos subyacentes a la enfermedad alérgica, por ejemplo, cómo evitar los factores que desencadenan e influyen en la producción o reducen los niveles de IL-4, puede ser útil para los programas de prevención. ${ }^{6,7}$

La inflamación causada por reacciones alérgicas en las vías respiratorias superiores e inferiores se asocia con citocinas producidas por células Th2 como IL-4 e IL-5. IL-4 ayuda a la producción de $\mathrm{IgE}$ por las células $\mathrm{B}$ y regula la adhesión de moléculas de células vasculares a las células endoteliales, lo que ayuda aún más a la transmigración de eosinófilos a los tejidos. IL-5 actúa como factor estimulante de colonias de eosinófilos y ayuda a la proliferación y diferenciación de eosinófilos en los tejidos. ${ }^{8}$ Estudios anteriores indicaron que la IL-4 y su variante polimórfica de receptores aumentaban el riesgo de asma al interactuar con la exposición al humo y las mascotas. ${ }^{9}$

La patogenia de las enfermedades alérgicas que involucran múltiples citocinas, quimiocinas y sus correlaciones analizadas como la base de la terapia de fase efectora, como anti-IgE, esteroides, miméticos $\beta 2$, antagonistas del receptor de leucotrienos. ${ }^{7}$ La IL-4 son citocinas, fundamentales y desempeñan un papel esencial en el desarrollo de la alergia. ${ }^{9}$ La alteración de estos genes puede dar lugar a diferentes respuestas a diversas sustancias sensibilizantes. $.^{10} \mathrm{C}-590 \mathrm{~T}$ es un polimorfismo promotor de IL-4 que influye en la transcripción de varios genes de citocinas. Es necesario investigar sobre mediadores inmunológicos que intervienen en la fase de centización como la IL-4, que juega un papel importante como desencadenante de las células B en la producción de IgE y como factor de polarización de las células $\mathrm{T}$ frente a las células Th2. ${ }^{9,11,12}$

El desarrollo y la progresión de la RA fueron interacciones complejas entre genética, epigenética, medio ambiente y estilo de vida. Los individuos susceptibles pueden experimen- tar una nueva sensibilización a las proteínas del medio ambiente y la interacción de la susceptibilidad genética y los factores ambientales que juegan un papel en el desarrollo de estas enfermedades alérgicas que implican la producción de IL- $4 .{ }^{8}$

La investigación sobre la correlación entre los niveles de interleucina-4 (IL-4) y la sensibilización a alérgenos del medio ambiente necesita conocer la dirección y la magnitud de la correlación. Esta correlación tiene un papel importante en los mecanismos de prevención de enfermedades en la etapa de sensibilización que precede a la fase efectora con anticuerpos neutralizantes de IL-4. La investigación de la IL-4 y sus interacciones con los factores ambientales puede ayudar a crear un nuevo procedimiento para detectar factores genéticos, de prevención e intervención.

\section{Métodos}

Se trata de un estudio analítico descriptivo con métodos observacionales, con diseño de investigación transversal. Este estudio ha cumplido con el número de elegibilidad de la prueba ética: 550 / EP-FKIK UMY / VII / 2013 por el comité de ética. Se calculó el tamaño de la muestra, según la fórmula de Slovin para la investigación transversal:

Donde:

$$
n=Z^{2} p(1-q) / d^{2}
$$

$Z=1.96$

$\mathrm{p}=$ prevalencia de incidencia de RA de la literatura

$\mathrm{d}=$ precisión absoluta

Con la cual se obtuvo una muestra mínima de 35.

Los criterios de inclusión en este estudio fueron pacientes con manifestaciones clínicas de RA que estaban dispuestos a cooperar y tenían una puntuación para los síntomas de RA basada en un cuestionario. Se excluyó de este estudio a los pacientes que estaban tomando antihistamínicos, con alteraciones dermatográfícas y padeciendo de enfermedades comórbidas. La recopilación de datos se llevó a cabo seleccionando a los encuestados con RA que cumplían con los criterios de inclusión y exclusión, respondiendo al consentimiento de los encuestados con consentimiento informado, luego de realizar una prueba de punción cutánea realizada en PKU Muhammadiyah Gamping Hospital y probando interleucina-4 (IL-4) niveles en el Laboratorio Biomédico de la Universidad Muhammadiyah Yogyakarta.

La prueba de punción cutánea se realizó analizando diez tipos de alérgenos, tres alérgenos inhalantes (polvo doméstico, algodón, plumas de pollo) y los otros alérgenos se ingestan, con aguja estéril roma. Se examinó el perfil de citocinas del suero sanguíneo para determinar los niveles de IL-4 mediante el método ELISA. La variable independiente en este estudio fue la sensibilización a alérgenos en pacientes con RA, mientras que la variable dependiente en este estudio 
fue la expresión de IL-4. El análisis de datos de este estudio utilizó el examen de datos de normalidad con histograma y prueba estadística de Spearman.

\section{Resultados}

Hubo 37 pacientes con RA que se convirtieron en encuestados de este estudio. Se dividió en 26 estudiantes mujeres (70.3\%) y 11 estudiantes hombres $(29.7 \%)$ con quejas de secreción nasal, estornudos y congestión nasal. Los resultados de la prueba de expresión de IL- 4 tuvieron un promedio de $22,48 \mathrm{pg} / \mathrm{mL}$, los valores normales son $0-65.18 \mathrm{pg} / \mathrm{mL}$, la distribución de datos examinada de normalidad con el histograma, mostró la tendencia inclinada hacia la izquierda, concluyó la distribución de datos anormal (Figura 1).

En los resultados de la evaluación de la sensibilidad a los alérgenos se puede observar que la positividad de alérgenos del 83,8 por ciento de los encuestados sensibilizados con el polvo doméstico como Aero alérgeno se muestra en el Cuadro 1.

Los datos de sensibilidad alérgica y expresión de IL-4 analizados con la prueba de correlación de Spearman mostraron el valor de $\mathrm{p}$ de 0.985 ( $\mathrm{p}>0.05$ ), indica que no hubo correlación entre la sensibilidad al alérgeno por inhalación y la expresión de IL-4. Tiene una dirección de correlación negativa y una fuerza de correlación muy débil de $0,003(0,00-$ 0,199), que se muestra en el Cuadro 2.

\section{Discusión}

En este estudio no hubo correlación significativa entre la sensibilidad al alérgeno probada con la expresión de IL-4 con un valor de $\mathrm{p}>0.05$ basado en la prueba de correlación de Spearman, y algunos alérgenos (camarón, atún, judías verdes) mostraron una dirección de correlación negativa. Este resulta-

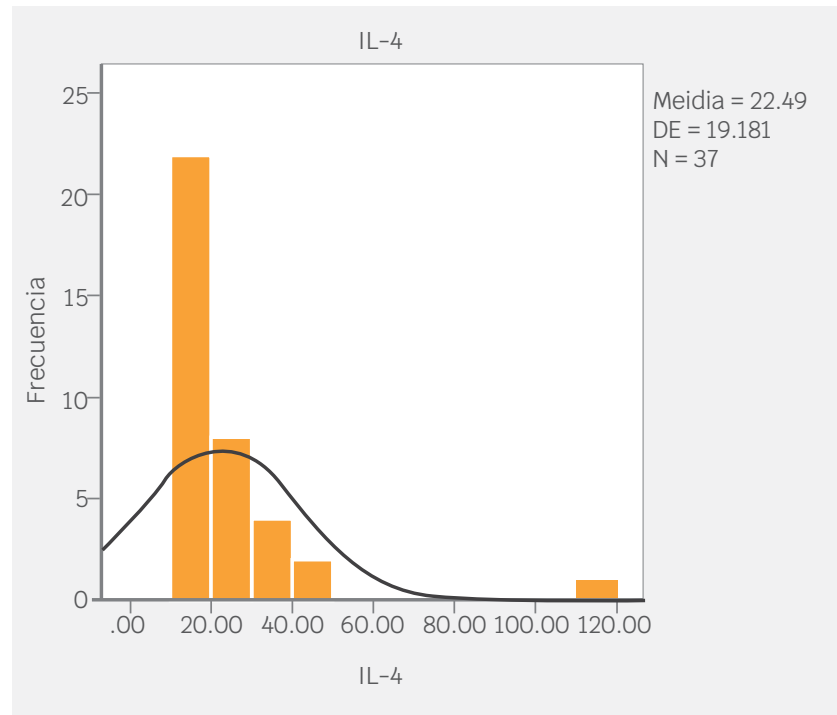

Figura 1. Distribución de datos de anomalías en la expresión de IL-4.

\begin{tabular}{|c|c|c|c|c|}
\hline Encuestado & Características & Resultado & $n$ & $\%$ \\
\hline \multirow{2}{*}{1} & \multirow{2}{*}{ Polvo doméstico } & Positivo & 31 & 83.8 \\
\hline & & Negativo & 6 & 16.2 \\
\hline \multirow{2}{*}{2} & \multirow{2}{*}{ Algodón } & Positivo & 15 & 40.5 \\
\hline & & Negativo & 22 & 59.5 \\
\hline \multirow{2}{*}{3} & \multirow{2}{*}{ Plumas de pollo } & Positivo & 11 & 29.7 \\
\hline & & Negativo & 26 & 70.3 \\
\hline \multirow{2}{*}{4} & \multirow{2}{*}{ Camarones } & Positivo & 10 & 27.0 \\
\hline & & Negativo & 27 & 73.0 \\
\hline \multirow{2}{*}{5} & \multirow{2}{*}{ Atún } & Positivo & 6 & 16.2 \\
\hline & & Negativo & 31 & 83.8 \\
\hline \multirow{2}{*}{6} & \multirow{2}{*}{ Yema } & Positivo & 16 & 43.2 \\
\hline & & Negativo & 21 & 56.8 \\
\hline \multirow{2}{*}{7} & \multirow{2}{*}{ Maní } & Positivo & 18 & 48.6 \\
\hline & & Negativo & 19 & 51.4 \\
\hline \multirow{2}{*}{8} & \multirow{2}{*}{ Clara de huevo } & Positivo & 14 & 37.8 \\
\hline & & Negativo & 23 & 62.2 \\
\hline \multirow{2}{*}{9} & \multirow{2}{*}{ Té } & Positivo & 12 & 32.4 \\
\hline & & Negativo & 25 & 67.6 \\
\hline \multirow{2}{*}{10} & \multirow{2}{*}{ Chocolate } & Positivo & 11 & 29.7 \\
\hline & & Negativo & 26 & 70.3 \\
\hline
\end{tabular}

do similar al informe de Valatabar et al. que mostró rs20541 en el CR de IL-13 y rs2243250 en la región promotora de IL-4 no se asoció con el riesgo de RA en adolescentes en el noroeste de Irán. ${ }^{13} \mathrm{Si}$ bien el informe Tyurin de 2017 también informó sobre pacientes con RA durante todo el año, hubo una correlación negativa entre las concentraciones séricas de IL-4 y los niveles de TGF- $\beta(r=-0,49, p=0,026)$ y hubo una correlación negativa entre la IgE sérica total niveles y lagartos IL-10. $(r=-0.63, p=0.002)$ у TGF- $\beta(r=-0.49$, $\mathrm{p}=0.01) .{ }^{14}$ Un estudio en población china también informó que el polimorfismo de IL-13 e IL4RA puede no tener una asociación significativa con los ácaros sensibilizados. ${ }^{15}$

El resultado de este estudio difiere del presente estudio que al comparar los pacientes en el grupo de casos con el grupo control, se exhibieron diferencias significativas entre el genotipo -590CT y el riesgo de RA en niños. Se ha demostrado que es probable que los polimorfismos del gen IL4 estén implicados en el desarrollo del asma, la RA y la regulación de la 


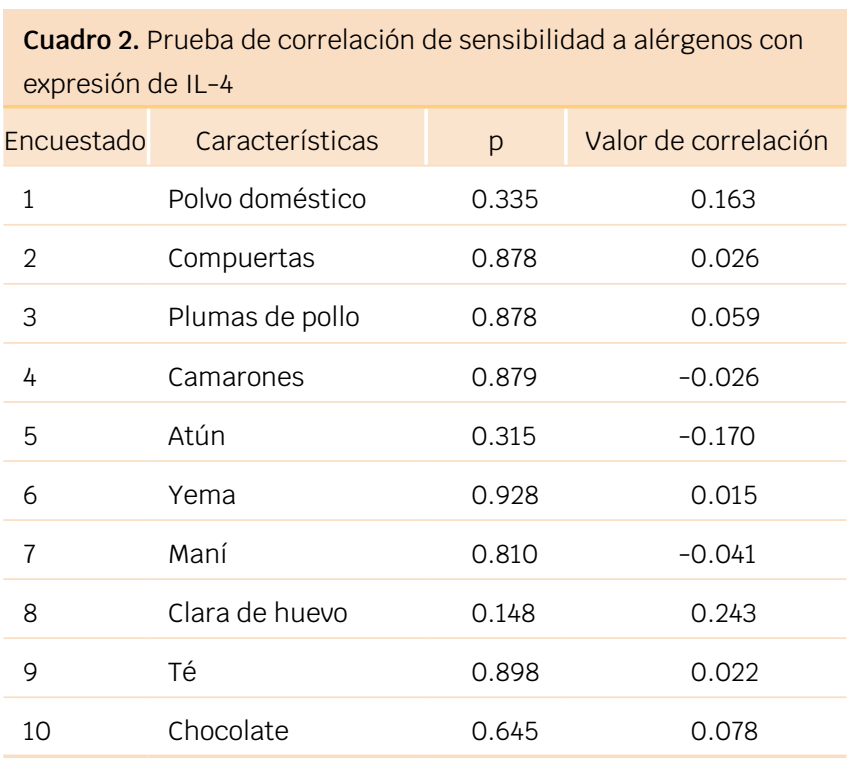

IgE sérica total. ${ }^{16}$ Otro estudio realizado por Narozna B, 2016, indicó que las variantes polimórficas en los genes de la cadena IL-4 e IL-4Ra podrían conferir susceptibilidad y modular la gravedad de la atopía y el asma en una población caucásica. ${ }^{17}$

IL-4 es importante en el desarrollo de enfermedades alérgicas debido a su efecto sobre las células $\mathrm{T}$ colaboradoras, y estimula directamente a las células B para que produzcan IgE e IgG4. La exposición a alérgenos en un cierto límite de concentración activa células Th2 específicas en la mucosa nasal de pacientes con RA, luego estimula la expresión aumentada de mRNA de citocinas Th2, especialmente IL-4 y reclutamiento de eosinófilos en el tracto respiratorio. En este estudio, la expresión de IL-4 no se correlacionó significativamente con la sensibilidad al alérgeno posiblemente debido a 1) La sensibilidad a los alérgenos es una interacción compleja entre el alérgeno y el huésped influenciada por factores genéticos y barreras físicas, químicas e inmunológicas. ${ }^{18}$
La susceptibilidad genética y los factores ambientales fueron cuestiones fundamentales en la patología de la RA. En este estudio, la mayoría de los tipos de sensibilización a los alérgenos inhalados fueron el polvo doméstico con un 83.8 \%. Estos resultados son similares a un estudio en Hong Kong que informó que los tipos más comunes de alérgenos en pacientes con rinitis crónica eran los ácaros del polvo doméstico (63\%), las cucarachas $(23 \%)$ y las escamas de piel de gato $(14 \%){ }^{19}$

Estos resultados son más pequeños que el estudio realizado por Navarro-Locsin en 2018, donde los alérgenos de interior más comunes en quienes padecen rinitis en Filipinas son los ácaros del polvo doméstico (HDM) Dermatophagoides pteronyssinus (97.4\%) y Dermatophagoides farinae $(95.8 \%)$, seguida de las cucarachas $(80.1 \%)$ y las setas $(72.8 \%) .{ }^{20}$ Por el contrario, los resultados del informe de sensibilización fueron más altos que los informados (Oncham S, 2018), donde los resultados de reactividad de alérgenos más inhalados en la población alérgica en Tailandia fueron ácaros con $50.1 \%$ de Dermatophagoides pteronyssinus y $32 \%$ de Dermatophagoides farinae, mientras que $31.5 \%$, polvo doméstico. ${ }^{21}$

La creciente prevalencia de enfermedades alérgicas en todo el mundo se asocia con predisposición genética, eventos epigenéticos y exposición ambiental, la sensibilización a proteínas extrañas del medio ambiente varía según las regiones afectadas por el clima, la urbanización y el estilo de vida. $^{22,23}$

\section{Conclusión}

No hubo correlación significativa entre la expresión de IL-4 y la sensibilización al alérgeno en pacientes con RA, varios alérgenos tienen una correlación negativa, y la mayor sensibilidad al alérgeno fue el polvo doméstico $(83.8 \%)$.

\section{Reconocimiento}

Gracias a LP3M UMY que financió esta investigación.

\section{Referencias}

1. Nolte $\mathrm{H}$, Nepper-Christensen S, Backer V. Unawareness and undertreatment of asthma and allergic rhinitis in a general population. Respir Med. 2006;100(2):354-362. DOI 10.1016/j.rmed.2005.05.012

2. Katelaris CH, Lee BW, Potter PC, Maspero JF, Cingi C, Lopatin A, et al. Prevalence and diversity of allergic rhinitis in regions of the world beyond Europe and North America. Clin Exp Allergy. 2012;42(2):186207. DOI: 10.1111/j.1365-2222.2011.03891.x

3. Migueres M, Dávila I, Frati F, Azpeitia A, Jeanpetit $Y$, LhéritierBarrand $M$, et al. Types of sensitization to aeroallergens: definitions, prevalences and impact on the diagnosis and treatment of allergic respiratory disease. Clin Transl Allergy. 2014;4:16. DOI: 10.1186/20457022-4-16
4. Bousquet J, Khaltaev N, Cruz AA, Denburg J, Fokkens WJ, Togias A, et al. Allergic Rhinitis and its Impact on Asthma (ARIA) 2008 Update (in collaboration with the World Health Organization, GA(2)LEN and AllerGen). Allergy. 2008;63(Suppl 86):S8-S160. DOI:10.1111/j.139899995.2007.01620.x

5. Brożek JL, Bousquet J, Baena CE, Bonini S, Canonica GW, Casale TB, et al. Allergic Rhinitis and its Impact on Asthma (ARIA) guidelines: 2010 revision. J Allergy Clin Immunol. 2010;126(3):466-476. DOI: 10.1016/j.jaci.2010.06.047

6. Kırmaz C, Bayrak P, Yıl-maz O, Yüksel H. Effects of glucan treatment on the Th1/Th2 balance in patients with allergic rhinitis: a doubleblind placebo-controlled study. Eur Cytokine Netw. 2005;16(2):128134. 
7. Albrecht M, Dittrich AM. Cytokines in sensitization to aeroallergens. Allergol Select. 2018;2(1):94-100. DOI: 10.5414/ALX1480E

8. Dittrich AM, Chen HC, Xu L, Ranney P, Connolly S, Yarovinsky TO, et al. A new mechanism for inhalational priming: IL-4 bypasses innate immune signals. J Immunol. 2008;181:7307-7315. DOI: 10.4049/ jimmunol.181.10.7307

9. Li L, LiY, ZengX, Li J, DuXY. Role of interleukin-4 genetic polymorphisms and environmental factors in the risk of asthma in children. Genet Mol Res. 2016;15(4):534-543. DOI: 10.4238/gmr15048873

10. Borish LC, Nelson HS, Corren J, Bensch G, Busse WW, Whitmore JB, et al. Efficacy of soluble IL-4 receptor for the treatment of adults with asthma. J Allergy Clin Immunol. 2001;107(6):963-970. DOI: 10.1067/ mai.2001.115624

11. Wenzel S, Wilbraham D, Fuller R, Getz-Burmesiter E, Longphre M. Effect of an interleukin-4 variant on late phase asthmatic response to allergen challenge in asthmatic patients: results of two phase 2a studies. Lancet. 2007;370(9596):1422-1431. DOI: 10.1016/S01406736(07)61600-6

12. Daniel WW. Biostatistics: a foundation for analysis in the health sciences. EEUU: John Wiley \& Sons; 1999.

13. Valatabar N, Hosseinpourfeizi M, Safaralizadeh R, Shabestari MS. Relationships between IL-13 and IL-4 genotypes and aeroallergens with risk of allergic rhinitis in Iranian-Azeri. Pediatr Allergy Immunol Pulmonol. 2020;33(1):33-38. DOI: 10.1089/ped.2019.1099

14. Tyurin YA, Lissovskaya SA, Fassahov RS, Mustafin IG, Shamsutdinov AF, Shilova MA, et al. Cytokine profile of patients with allergic rhinitis caused by pollen, mite, and microbial allergen sensitization. J Immunol Res. 2017;2017:1-8. DOI: 10.11155/2017/3054217

15. Lu M-P, Chen R-X, Wang $M-L$, Zhu $X-J$, Zhu L-P, Yin $M$, et al. Association study on IL4, IL13 and IL4RA polymorphisms in mitesensitized persistent allergic rhinitis in a Chinese population. PLoS One. 2011;6(11):e27363. DOI: 10.1371/journal.pone.0027363
16. Al-Rawashdeh BM, Sadaalhanjori A, Ali E, Zihlif M. Association of IL-4 polymorphisms with allergic rhinitis in Jordania population. Medicina (Kaunas). 2020;56(4):179. DOI: 10.3390/ medicina56040179

17. Narozna B, Hoffmann A, Sobkowiak P, Schoneich N, Bręborowicz A, Szczepankiewicz A, et al. Polymorphisms in the interleukin 4 , interleukin 4 receptor and interleukin 13 genes and allergic phenotype: a case control study. Adv Med Sci. 2016;61(1):40-45. DOI: 10.1016/j.advms.2015.07.003

18. Korzycka-Zaborowska B, Zielińska-Bliźniewska H, Zaborowski A, Oiszewski J. Association of-590 C/T Il-4 gene promoter polymorphism with atopy in Polish patients with allergic rhinitis. J Allergy Disord Ther. 2015;2:100004. DOI: 10.24966/ADT-749X/100004

19. Yuen APW, Cheung S, Tang KC, Ho WK, Wong BYH, Cheaung ACS, Ho ACW. The skin prick test results of 977 patients suffering from chronic rhinitis in Hong Kong. Hong Kong Med J. 2007;13(2):131-136. Disponible en: https://www.hkmj.org/system/files/hkm0704p131.pdf

20. Navarro-Locsin CG, Lim-Jurado M. Aeroallergen sensitization and associated comorbid diseases of an adult Filipino population with allergic rhinitis. Asia Pac Allergy. 2018;8(3):e25. DOI: 10.5415/ apallergy.2018.8.e25

21. Oncham S, Udomsubpayakul U, Laisuan W. Skin prick test reactivity to aeroallergens in adult allergy clinic in Thailand: a 12-year retrospective study. Asia Pac Allergy. 2018;8(2):e17. DOI: 10.5415/ apallergy.2018.8.e17

22. Pawankar R, Canonica G, Holgate S, Lockey R, Blaiss, M. WAO White Book on Allergy; Allergy Organization: Milwaukee, WI, USA, 2011; Volume 3: 156-157.

23. Passali D, Cingi C, Staffa P, Passali F, Muluk NB, Bellussi, ML. The international study of the allergic rhinitis survey: outcomes from 4 geographical regions. Asia Pac J Allergy. 2018;8(1):e7. DOI: 10.5415/ apallergy.2018.8.e7

ORCID

Asti Widuri, 0000-0002-7558-8991 